\title{
Präferenzen der Proctodrilus-Arten (Lumbricidae: Oligochaeta) auf einem Hang des unteren Kokel-Hochlandes in Rumänien
}

\author{
N. HÖSER \\ Norbert Höser, Am Park 1, D-04603 Windischleuba, Germany. \\ E-Mail: norbert.hoeser@arcor.de
}

\begin{abstract}
Preferences of the Proctodrilus species (Lumbricidae: Oligochaeta) on a slope of the lower 'Kokel' uplands in Romania. On a slope in the 'Kokel' uplands in Romania (Transylvanian Piedmont at the foot of the eastern Carpathians) the earthworm species Proctodrilus tuberculatus (Černosvitov, 1935), P. thaleri Höser \& Zicsi, 2009 and P. opisthoductus Zicsi, 1985 were found at different points in the soil catena. The colluvial soil clearly has the distinctive characteristics of an inverse profile. P. tuberculatus occurs at the top of the slope and in the colluvial soil, while $P$. thaleri was found exclusively in the area at the foot of the slope towards the valley, in the section of greater colluvial horizon diversity. P. opisthoductus occurs towards the valley floor, in the relatively humus-rich mineral layer of the inverse profile. The pedological findings indicate that $P$. tuberculatus prefers the leached horizons of the lessived soils. Where $P$. thaleri occurs is a colluvial soil with a probable tendency to a soil-type interference of lessive soil and brown loam (terra fusca), where these two types are within one and the same soil profile. Possibly the sedimentary lessivage complexes of a terra fusca character (plasma flow) make it easier for the Proctodrilus species to move through the solum.
\end{abstract}

Keywords. Earthworms, slope soil catena, lessivage, Romania, endemic species, ecological vicariants, Proctodrilus

\section{EINLEITUNG}

E in Nachweis von Proctodrilus thaleri Höser \& Zicsi, 2009 bei Celei im Getischen Piedmont wurde anhand der pedologischen Befunde dem Habitat einer Pseudogley-Lessivé-Braunlehm-Interferenz zugeordnet (Höser 2013). Lessivé-Braunlehm-Interferenzen sind im warm-humiden Südosteuropa mit rezenten Lessivés vergesellschaftet (Reuter 1999). Regelmäßige Vergesellschaftungen von Bodentypen in der Hangcatena werden von der Hanggestalt, dem lokalen Grund- und Stauwasser und der Mächtigkeit und Art der Sedimentdecke bestimmt (Schmidt 1997). Der Fund von Celei führte zur Erwartung, südosteuropäische Hänge zu finden, auf denen $P$. tuberculatus und $P$. thaleri ökologisch vikariant an unterschiedlichen Stellen der Bodencatena leben. Ein derartiger Hang wurde im KokelHochland in Rumänien gefunden. Davon wird im Folgenden berichtet.

\section{Untersuchtes Gebiet}

Das untersuchte Hanggelände (440 - 520 m ü. $\mathrm{NN}$ ), das teilweise bewaldet ist, liegt $c a .2 \mathrm{~km}$ östlich von Betfalva / Beteşti (Kreis Harghita) im Großkokler-Homoroder Piedmont (Heltmann \& Servatius 1991) des östlichen Kokel-Hochlands in Rumänien. Es befindet sich an der östlichen Seite eines ungefähr in Nord-Süd-Richtung verlaufenden rechten Seitentals (Fâneaţa Îngustă) der Großen Kokel (Nagy-Küküllö, Târnava Mare).

Das Bodensubstrat besteht hier aus gelblichem Mergel und Löss, der tertiäre Schichten überlagert und verhüllt (Hauer \& Stache 1863). Die Böden des Gebietes sind teils mitteleuropäische Braunerden, zumeist aber (und in den mehr ebenen Lagen) fast skelettlose Lessivés und Lessivé-Pseudogleye (Ganssen \& Hädrich 1965, Stefanovits 1971, Conea et al. 1973). In Dellen und an Hangfüßen lagern mächtige bindige Kolluvien. 
Die mittlere Jahrestemperatur der Region beträgt $c a .9^{\circ} \mathrm{C}$, infolge der südexponierten Lage am wärmebegünstigten Kokeltal sicher lokal etwas mehr, der mittlere Jahresniederschlag 700 bis $800 \mathrm{~mm}$.

Das untersuchte Gelände liegt am Rand des Weinanbaugebiets und gehört zum Buchengebiet des rumänischen Karpatenvorlandes (Rubner \& Reinhold 1953); es liegt in der dakisch-karpatischen Buchenwaldzone (Duty 1985, Dierschke 1990).

\section{Das untersuchte Hanggelände}

Das untersuchte Hanggelände hat ein komplexes Profil, bestehend aus mehreren Segmenten. Im oberen Teil befindet sich ein SteildellenTalanfang (Ahnert 1999, S. 274). Diese Steildelle (Abb. 1) und das aufnehmende Tal Fâneaţa Îngustă sind nicht gleichsohlig. An mehreren Hangstellen wurden im Boden Tonhäutchen als Merkmale der Lessivierung gefunden.
Der Hangscheitel ist hier ein Plateau. Im bewaldeten Bereich dieses Hanggeländes herrscht Hainbuche (Carpinus betulus) vor, begleitet von Traubeneiche (Quercus petraea), Vogelkirsche (Cerasus avium) und Feldahorn (Acer campestre). Auf dem Hangplateau tritt auch spärlich Rotbuche (Fagus sylvatica) auf.

Augenscheinlich wächst im untersuchten Transekt das Lathyro-Carpinion Boşcaiu 1974, in dem Stellaria holostea und aus der transylvanischen „Carpinion“-Artengruppe Scilla bifolia und Dactylis polygama auffallen. Zwei regionaltypische Assoziationen (Kovács 2007) sind vertreten: Auf dem Hang überwiegt das Lathyro hallersteinii-Carpinetum Coldea 1975 mit Brachypodium sylvaticum, Mercurialis perennis, Crataegus monogyna und Ligustrum vulgare, auf dem Hangplateau das Carpino-Fagetum Paucă 1941 mit Aegopodium podagraria, Alliaria petiolata und Daphne mezerum. Der untere Teil des Geländes und die Sohle der Steildelle werden als Mähwiesen genutzt.

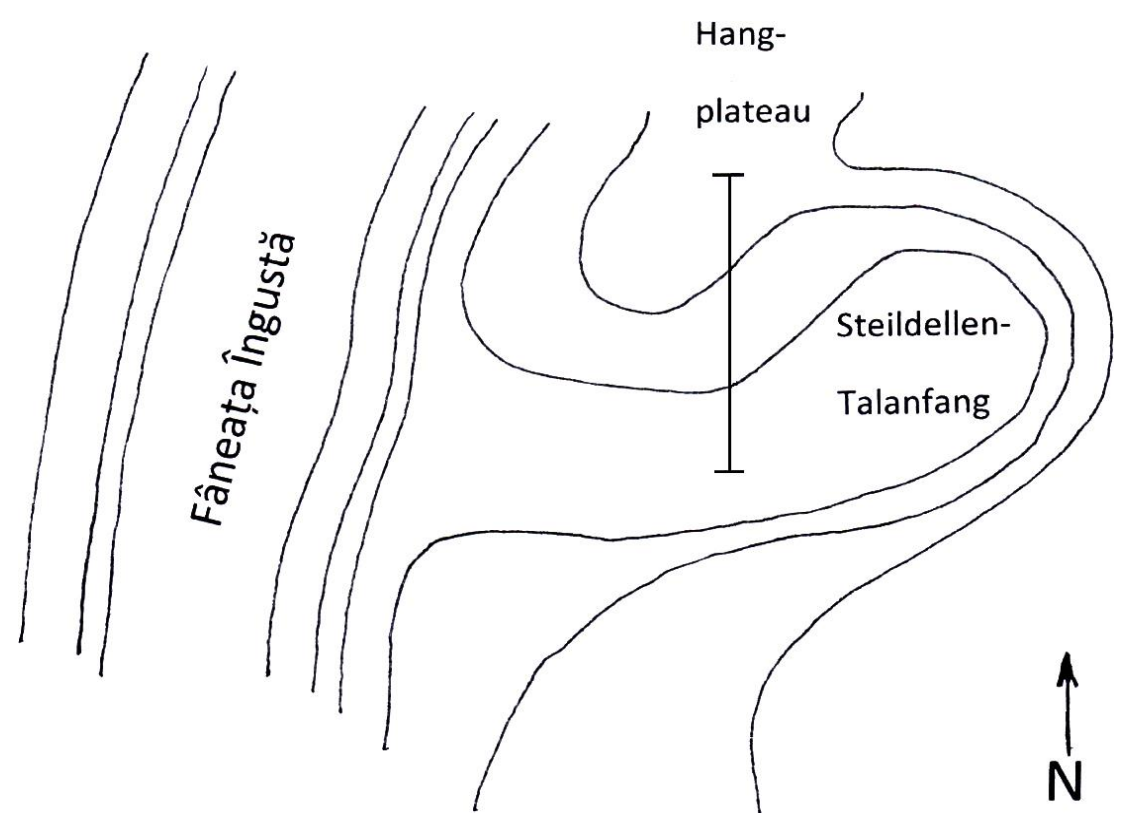

Abbildung 1. Lage des Transekts im Hanggelände des Steildellen-Talanfangs bei Betfalva. Schematischer Verlauf der Isohypsen. 


\section{MATERIAL UND METHODEN}

Die Regenwurmfauna wurde auf jeweils 0,25 $\mathrm{m}^{2}$ großen Untersuchungsflächen in mehreren Teilen des Hanggeländes erfasst. Im vorliegenden Beitrag vorgestellt werden die Ergebnisse vom Transekt, das entlang der Fall-Linie des südexponierten Hangs der Steildelle führt (Abb. 1, 2). Dieser Hang hat ein sigmoides Profil und ist mittelstark (ca. $15 \%$ oder 8 Grad) geneigt. Weitere Probeflächen im Hanggelände, sowohl zufällig verteilte als auch mehrere entlang eines anderen Transekts im unteren Geländeteil bis zum Bach (Fâneaţa Îngustă), dienten der Übersicht und Ergänzung.

Auf jeder Untersuchungsfläche von $0,5 \times 0,5$ $\mathrm{m}$ wurden bis in $0,5 \mathrm{~m}$ Tiefe alle Regenwürmer ausgegraben und in zweimaliger Durchsicht des ausgegrabenen Bodens von Hand ausgelesen. Für die Auswertung wurden ausschließlich die adulten Tiere herangezogen. Die taxonomische und zoogeographische Nomenklatur folgt Csuzdi \& Zicsi (2003), Csuzdi et al. (2011) und Pop et al. (2012). Konserviertes Material befindet sich in der Sammlung des Autors.

Jede beim Ausgraben hergestellte Schürfgrube diente der Bodenansprache. Korngradierungen und Feuchtestufen des Bodens wurden nach feldmethodischen Kriterien (Fiedler \& Schmiedel, 1973, Ad-hoc-AG Boden, 2005) eingeschätzt. Die genetisch-morphologische Horizontkennzeichnung des Bodens berücksichtigt die von Reuter (1999) begründete. Von einzelnen Standorten wurde der Glührückstand des Bodens bestimmt.

\section{ERGEBNISSE}

\section{Bodenprofile des Hangs der Steildelle}

Im Bodenprofil auf dem Plateau (Scheitel) des Hangwaldes folgt unter dem schwachen LHorizont, der überwiegend aus Hainbuchen- und Eichenblättern besteht, eine dünne Humusauflage (Of, Oh). An diese schließt sich ein 3-8 cm mächtiger dunkelbrauner, humoser, bindiger Mineralboden (Ah-Horizont) an. Dem folgt tonigschluffiger Lösslehm, oben hellbraun aufgehellt (Al- bzw. El-Horizont), unten mittelbraun und merklich mit Ton angereichert (Bt- bzw. It-Horizont).

Auf dem Mittelhang beträgt der Ah-Horizont 3 cm über geköpften Profilen, auf dem Unterhang 5 $\mathrm{cm}$. Am baumbestandenen Unterhang wird dieser Horizont von geröllhaltigem hellbraunem Lösslehm unterlagert, der ab ca. $20 \mathrm{~cm}$ Tiefe in einen Skelettboden übergeht.

Das Kolluvium des Hangfußes hat hangseitig den größten Feinsand-Anteil. Dort existiert unter dem mittelbraunen Boden ein in $36 \mathrm{~cm}$ Tiefe begrabener, mehr als $15 \mathrm{~cm}$ mächtiger dunkler Horizont des Inversprofils, der bis zur Sohlenmitte hin aushält und aufsteigt und dort schließlich zutage tritt. Infolgedessen zeigt sich im AHorizont der Wiese vom Hangfuß zur Sohlenmitte hin ein deutlicher Wechsel vom hell mittelbraunen, feinsandreichen Substrat des Schwemmfächers zum nahezu schwarzen, klebrigen Boden, der von höherem Anteil der Tonfraktion zeugt. In derselben Richtung nimmt die mittlere Bodenfeuchte zu, ebenso mit zunehmender Tiefe, so von erdfeucht zu gut durchfeuchtet, aber nicht nass. Jenseits der Sohlenmitte fallen die Tonhäutchen und der Wasserfilm des polyedrischen Gefüges stärker auf.

\section{Artenspektrum und Lebensformen der Regenwurmfauna}

Entlang des vorgestellten Transekts wurden 10 Arten gefunden (Abb. 1, Tabelle 1), von denen die Mehrheit zur endogäischen Lebensform gehört. Als Vertreter der epigäischen Arten wurden nur D. octaedra und L. rubellus angetroffen. Eine tiefgrabende (anözische) Art wurde gefunden, nämlich Fitzingeria platyura. In größter Stetigkeit kommen die endogäischen Arten Ap. rosea, Ap. georgii und $O$. lacteum vor. Auffällig ist, dass $A p$. caliginosa fehlt. 


\section{Verteilung der Regenwurmarten am Hang}

Unter dem nahezu geschlossenen Baumbestand des südexponierten Hangs der Steildelle konnten weder epigäische Arten noch der epiendogäische L. rubellus nachgewiesen werden. Am Fuße dieses Hangsegments beginnt der sonnenexponierte Bereich der Steildellen-Wiese. Im anschließenden Teil dieser Wiese leben die epigäische D. octaedra und der L. rubellus auf der Steildellen-Sohle im Einflussbereich des Hangzugwassers. Andererseits treten die beiden Arten außerhalb des hier vorgestellten Transekts auch im nordwestexponierten Boden des Hangwaldes spärlich auf.

O. lacteum kommt in allen Teilen des Hangprofils vor. Die gefundenen Vertreter der Gattung Octodrilus wurden in unterschiedlichen Relief- positionen des Hangs angetroffen, nicht jedoch im vielschichtigen Bereich des Schwemmfächers. $F$. platyura bevorzugt im Transekt die weit entwickelten, tiefgründigen Bodenprofile auf dem Hangscheitel. A. georgii und A. rosea sind auf die Wiesenböden des Hanggeländes beschränkt. $P$. tuberculatus lebt auf dem Hangscheitel und im Kolluvium, während $P$. thaleri ausschließlich im talseitigen Bereich des Hangfußes, im Abschnitt größter Schichtenvielfalt, gefunden wurde.

Am artenreichsten ist die Regenwurmfauna auf den nahezu ebenen Wiesen-Standorten des Steildellen-Talanfangs. Ihre Individuendichte ist im Einflussbereich des Hangzugwassers und am schattigen Fuß des nicht abgebildeten, nordexponierten Gegenhangs der Steildelle größer als am Hang, der in Abb. 2 vorgestellt wird. Das betrifft vor allem die epigäischen Arten.

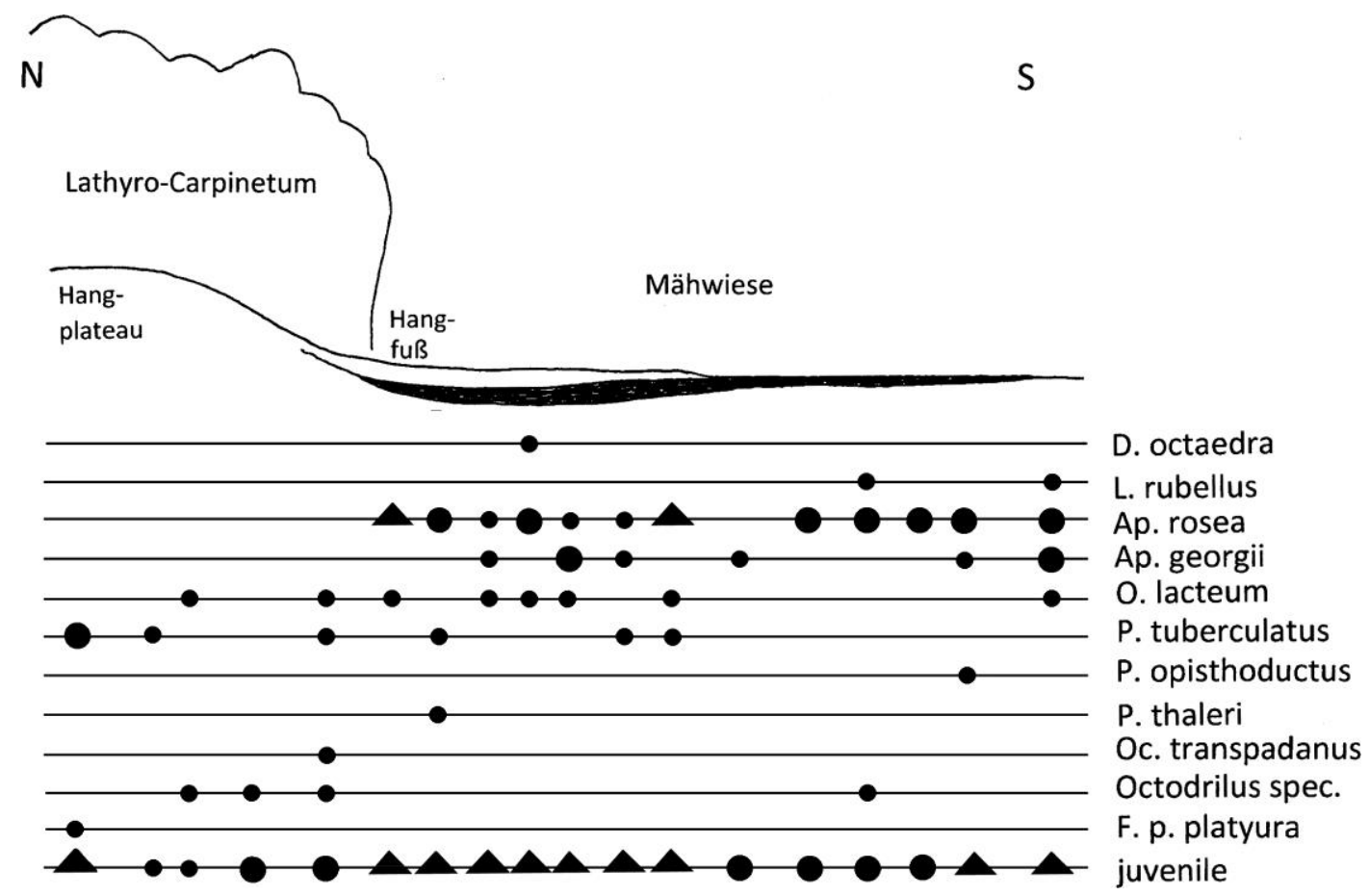

Abbildung 2. Regenwurmfauna der untersuchten Bodenstandorte im Hang-Transekt bei Betfalva. Mit dunklem Horizont des Inversprofils. Die Punkte bzw. Dreiecke repräsentieren die Individuendichte der adulten Regenwürmer und der Juvenilen in drei Größenklassen: • 1-3, • 4-10, $\boldsymbol{\Delta}>10$ Individuen auf 0,25 $\mathrm{m}^{2}$ untersuchter Fläche. Transektlänge $\mathrm{ca} .80 \mathrm{~m}$. 
Tabelle 1. Übersicht über die am Hang bei Betfalva / Beteşti gefundenen Regenwurmarten mit Angaben zum Verbreitungstyp (Csuzdi et al. 2011, Pop et al. 2012), zur ökologischen Lebensform (Bouché 1977) und maximalen und durchschnittlichen Individuendichte (Mittelwert \pm Standardfehler).

\begin{tabular}{|c|c|c|c|c|}
\hline \multirow[t]{2}{*}{ Art im Transekt (Abb. 2) } & \multirow{2}{*}{$\begin{array}{l}\text { Verbreitungs- } \\
\text { typ }\end{array}$} & \multirow[t]{2}{*}{ Lebensform } & \multicolumn{2}{|c|}{$\begin{array}{l}\text { Individuendichte } \\
\text { Adulte auf } 0,25 \mathrm{~m}^{2}\end{array}$} \\
\hline & & & maximal & Mittelwert \\
\hline Aporrectodea georgii (Michaelsen, 1890) & atlanto-mediterran & endogäisch & 5 & $0,9 \pm 0,35$ \\
\hline Aporrectodea rosea (Savigny, 1826) & peregrin & endogäisch & 12 & $4,2 \pm 0,96$ \\
\hline Dendrobaena octaedra (Savigny, 1826) & peregrin & epigäisch & 1 & $<0,1$ \\
\hline Fitzingeria platyura (Fitzinger, 1833) & $\begin{array}{l}\text { zentraleuropäisch } \\
\text { montan }\end{array}$ & anözisch & 1 & $<0,1$ \\
\hline Lumbricus rubellus Hoffmeister, 1843 & peregrin & epigäisch & 3 & $0,2 \pm 0,17$ \\
\hline Octolasion lacteum (Örley, 1881) & peregrin & endogäisch & 3 & $0,7 \pm 0,22$ \\
\hline Octodrilus transpadanus (Rosa, 1884) & trans-ägäisch & endogäisch & 1 & $<0,1$ \\
\hline Proctodrilus opisthoductus Zicsi, 1985 & zentraleuropäisch & endogäisch & 2 & $<0,1$ \\
\hline Proctodrilus tuberculatus (Černosvitov, 1935) & trans-ägäisch & endogäisch & 6 & $0,7 \pm 0,34$ \\
\hline Proctodrilus thaleri Höser \& Zicsi, 2009 & karpatisch-endemisch & endogäisch & 2 & $<0,1$ \\
\hline
\end{tabular}

\section{Bodenstandorte der Proctodrilus-Arten}

$P$. tuberculatus besiedelt den Lessivé auf dem nahezu ebenen Hangscheitel (= Hangplateau) und das anschließende Kolluvisol des Unterhangs, des Hangfußes und der anschließenden Fußfläche. An diesen Stellen wurde die Art ausschließlich im hell- bis mittelbraunen, teils dunkelgrauen, bindigen Mineralboden gefunden. So tritt sie auf dem Hangscheitel im obersten Dezimeter dieses Mineralbodens auf, im El-Horizont, der den dunklen Mull-Horizont (Ah) unterlagert, während sie am Hangfuß im grobkörnigeren Teil der geschichteten Schwemmfächerdecke angetroffen wird.

Zwei adulte $P$. thaleri konnten im vielfältig geschichteten Kolluvium des Hangfußes entdeckt werden, so im oberflächennahen mittelbraunen Boden, der den begrabenen, nahezu schwarzen Horizont des Bodenprofils überlagert. Dieser Standort befindet sich am talseitigen Rand der Schwemmfächerdecke, wo der horizontale Gradient des Ton- und Schluff-Anteils sein Maximum erreicht.

In der Sohlenmitte tritt im dunklen, aus dem Inversprofil aufgestiegenen, relativ humosen Mineralboden (Glühverlust 15,0 \%) P. opisthoductus auf.

\section{DISKUSSION}

Die Regenwurmarten des untersuchten Hangs sind ebenfalls im Artenspektrum der Fauna siebenbürgischer Flussauen zu finden (Höser 2003, 2011), auch Octodrilus transpadanus (Erratum in Höser 2000, 2003: syn. Oc. exacystis).

Die vertikalen und horizontalen Feuchtegradienten der untersuchten Hangcatena sind wahrscheinlich geprägt vom Hangzugwasser, das die Bodengenese, z.B. auch die Lessivierung, erheblich zu intensivieren vermag (Blume 1973) und die feuchteren, von epigäischen Arten $(D$. octaedra, $L$. rubellus) angezeigten Geländebereiche des Hangs und der Steildellen-Sohle verursacht.

Nach bisherigen Erfahrungen tritt $P$. tuberculatus auf erosionsdisponierten Bodenstandorten der Hanglagen und flussnahen Auenböden auf (Höser 2008, 2012), die von intensiver Lessivierung geprägt wurden. Er bevorzugt anscheinend eluviale Horizonte. Auch im untersuchten Transekt Betfalva trägt das Hangplateau diese Merkmale der typischen Lessivierung und auf dem zugehörigen Unterhang und Talboden gibt es als lessivierte Abkömmlinge die geköpften Profile bzw. kolluvialen Sedimente. Folgerichtig 
konnte an diesen drei Hangstandorten $P$. tuberculatus nachgewiesen werden (Abb. 2).

Im talseitigen Schwemmfächer-Teil des Hangfußes bei Betfalva vermuten wir als Resultat kolluvialer Sedimentation besondere Standortbedingungen zugunsten von $P$. thaleri: Im Zuge des Hangabtrags erfolgte dort wahrscheinlich an sedimentär bedingten dichteren Bodenschichten eine Tonanreicherung, die feldmethodisch gut nachgewiesen ist. Möglicherweise enthält das dort abgelagerte Maximum von Ton und Schluff eine relevante Menge von Schlämmstoffablagerungen, die aus dem Braunlehmgefüge stammen, das sowohl aus den im Löss stets vorgebildet enthaltenen Resten alter Bodendecken (Kubiena 1956) als auch aus der in Südosteuropa häufigen Vergesellschaftung des Lessivé mit Braunlehm (Reuter 1964) stammen kann. Braunlehm entwickelte sich in hügeligen Gebieten zumeist auf Verebnungen und breiteren Sattellagen (Kösel 2008). Also nehmen wir an, dass am Hangfuß bei Betfalva ein Kolluvisol mit Tendenz zur LessivéBraunlehm-Interferenz (Reuter 1999) vorliegt. Zu diesen Deutungen passen auch die pedologischen Befunde der von $P$. thaleri besiedelten Verebnung eines Hangs bei Celei (Höser 2013) und die festgestellte Präferenz der Art für Grenzen von feinkörnigeren Schichten (Höser \& Zicsi 2009).

Unsere Beobachtungen lassen vermuten, dass ein spezifischer Zusammenhang der ProctodrilusGruppe mit jenen besonderen Böden besteht, die sich durch stark bewegliche Feinsubstanz und Fließstrukturen der Feinsubstanz auszeichnen. In Lössböden tritt diese Feinsubstanz als Braunlehmteilplasma auf, so in den Leitbahnen im Braunerde-Grundgefüge des Lessivé oder, wenn dieses Grundgefüge durch Staunässe verloren gegangen ist, in unregelmäßigen Flecken im Pseudogley und Stagnogley (Kubiena 1956). An die Fließstrukturen des Lessivé, so bevorzugt in dessen eluvialem Horizont, ist offenbar P. tuberculatus gebunden, während $P$. antipai den beiden anderen Böden nahe steht. Die Befunde bei Celei (Höser 2013) und die vorliegenden von Betfalva sprechen dafür, dass $P$. thaleri mehr als die vorgenannten beiden Arten dem vom Braunerde-
Grundgefüge freien, feindispersen Gefügeplasma des Braunlehms zugeneigt zu sein scheint. Möglicherweise erleichtern die Schlämmstoffkomplexe von Braunlehmcharakter den Proctodrilus-Arten, sich im Solum zu bewegen.

Dank - Herr Prof. Dr. András Zicsi (†) überprüfte einige Artbestimmungen, Herr Prof. Dr. Csaba Csuzdi gab Hinweise zum Beitrag, Herr Michael Höser übernahm die technische Assistenz im Freiland und Herr Brian Hillcoat (Berlin) die Übersetzung ins Englische.

\section{LITERATUR}

AD-HOC-AG BODEN des Bund-Länder-Ausschusses Bodenforschung (2005): Bodenkundliche Kartieranleitung (KA 5). 5. Auflage, Hannover, 438 pp.

AHNERT, F. (1999): Einführung in die Geomorphologie. 2. Auflage. Verlag Eugen Ulmer, Stuttgart, $440 \mathrm{pp}$.

BluME, H.-P. (1973): Genese und Ökologie von Hangwasserböden. In. SCHLICHTING, E. \& Schwertmann, U. (Eds.) Pseudogley \& Gley. Verlag Chemie, Weinheim, p. 187-194.

BouCHÉ, M. B. (1977): Stratégies lombriciennes. In. LOHM, U. \& PERSSON, T. (Eds.) Soil organisms as components of ecosystems. Proc. VI. Int. Soil Zoology Colloquium. Ecological Bulletins (Stockholm), 25: 122-132.

Conea, A., OANCEA, C., PopovăȚ, A., RAPAPORT, C. \& VINTILĂ, I. (1973): Comparative Study of Planosols in Romania. In. SCHLICHTING, E. \& Schwertmann, U. (Eds.) Pseudogley \& Gley. Verlag Chemie, Weinheim, p. 323-331.

CsuZDI, Cs. \& ZICSI, A. (2003): Earthworms of Hungary (Annelida: Oligochaeta, Lumbricidae). In. Csuzdi, Cs. \& MAHunKA, S. (Eds.) Pedozoologica Hungarica 1, Hungarian Natural History Museum, Budapest, $271 \mathrm{pp}$.

Csuzdi, Cs., PoP, V. V. \& PoP, A. A. (2011): The earthworm fauna of the Carpathian Basin with new records and description of three new species (Oligochaeta: Lumbricidae). Zoologischer Anzeiger, 250(1): 2-18.

DIERSCHKE, H. (1990): Species-rich beech woods in mesic habitats in central and western Europe: a regional classification into suballiances. Vegetatio, 87: $1-10$. 
DuTY, J. (1985): Die Fagus-Sippen Europas und ihre geographisch-soziologische Korrelation zur Verbreitung der Assoziationen des Fagion s.l. Vegetatio, 59: 177-184.

FIEDLER, H. J. \& SCHMIEDEL, H.(1973): Methoden der Bodenanalyse. Band 1. Feldmethoden. Theodor Steinkopff, Dresden, 239 pp.

GANSSEN, R. \& HÄDRICH, F. (1965): Atlas zur Bodenkunde. Bibliographisches Institut, Mannheim, $85 \mathrm{pp}$.

HAUER, F. VON \& STACHE, G. (1863): Geologie Siebenbürgens. Nach den Aufnahmen der K.K. Geologischen Reichsanstalt und literarischen Hülfsmitteln zusammengestellt. Wilhelm Braumüller, Wien, 636 pp.

Heltmann, H. \& Servatius, G. (1991): Die naturräumliche Gliederung Siebenbürgens. In. HELTMANN, H. (Ed.) Naturwissenschaftliche Forschungen über Siebenbürgen IV. Siebenbürgisches Archiv, 25: $91-120$.

HÖSER, N. (2000): Die Verteilung der azonalen Elemente der Regenwurmfauna in Auenquerschnitten von Kokel und Mieresch. In. HELTMANN, H. \& VON KILLYEN, H. (Eds.) Naturwissenschaftliche Forschungen über Siebenbürgen VI. Siebenbürgisches Archiv, 36: 219-232.

HÖSER, N. (2003): Die Verteilung der Regenwürmer in der Aue des Mieresch (Siebenbürgen, Banat, Rumänien). Verhandlungen der Zoologisch-Botanischen Gesellschaft in Österreich, 140: 99-116.

HÖSER, N. (2008): Die Regenwürmer Proctodrilus tuberculatus (Cernosvitov, 1935) und Proctodrilus antipai (Michaelsen, 1891) als Indikatoren der fossilen Oberflächengliederung der Flussaue (Oligochaeta: Lumbricidae). Hercynia N.F., 41 (2): 263-272.

HöSER, N. (2011): Die Regenwurmfauna (Oligochaeta: Lumbricidae) der Aue der Großen Kokel als Abhängige der Böden im Flusseinzugsgebiet und der Auengradienten. Opuscula Zoologica Budapest, 42 (1): 23-34.

HÖSER, N. (2012): Regenwürmer (Oligochaeta: Lumbricidae) in der Hangcatena. Hercynia N.F., 45 (2): 193-208.

HÖSER, N. (2013): Proctodrilus thaleri (Oligochaeta: Lumbricidae) auf einem Hang in Oltenien. Opuscula Zoologica Budapest, 44 (1): 85-90.
HÖSER, N. \& ZICSI, A. (2009): Eine neue ProctodrilusArt (Oligochaeta: Lumbricidae) und ihr spezifischer Ökotop. Contributions to Natural History, 12 (2): 651-658.

KÖSEL, M. (2008): Landschafts- und Bodenentwicklung auf der Schwäbischen Alb. In. TERHORST, B. \& KÖSEL, M. (Eds.) Exkursionsführer 27. Jahrestagung des AK Paläopedologie der DBG 1.3. Mai 2008 in Tübingen, p. 51-77.

KovÁCS, J.A. (2007): Data to the vegetation biology and coenological relations of Allium ursinum L. stands in Eastern Transylvania. Kanitzia, 15: 6376.

KubIENA, W.L. (1956): Zur Mikromorphologie, Systematik und Entwicklung der rezenten und fossilen Lößböden. Eiszeitalter und Gegenwart, 7: 102-112 (und 4 Tafeln).

PoP, V. V., PoP, A. A. \& CsUZDI, Cs. (2012): An annotated checklist of the Romanian earthworm fauna (Oligochaeta: Lumbricidae). In. PAVLIČEK, T., CARDET, P., Csuzdi, Cs., LE BAYON, R.-C. \& GoBAT, J.-M. (Eds.) Advances in Earthworm Taxonomy V (Annelida: Oligochaeta). Proceedings of the $5^{\text {th }}$ International Oligochaeta Taxonomy Meeting Beatenberg, Switzerland, 11-15 April, 2011. Zoology in the Middle East Supplementum, 4: 59-70.

REUTER, G. (1964): Vergleichende Untersuchungen an lessivierten Böden in verschiedenen Klimagebieten. 8th International Congress of Soil Science, Bukarest, 5: 723-732.

REUTER, G. (1999): Statement zur Lessivé-Problematik in Mitteleuropa. Mitteilungen der Deutschen Bodenkundlichen Gesellschaft, 91: 1065-1068.

RUBNER, K. \& REINHOLD, F.(1953): Das natürliche Waldbild Europas als Grundlage für einen europäischen Waldbau. Paul Parey, Hamburg und Berlin, 288 pp.

SCHMIDT, R. (1997): Grundsätze der Bodenvergesellschaftung. In. BLUME, H.-P., FELIX-HENNINGSEN, P., Fischer, W.R., Frede, H.-G., Horn, R. \& STAHR, K. (Eds.) Handbuch der Bodenkunde. 3. Ergänzungslieferung, Ecomed Verlagsgesellschaft, Landsberg/ Lech, Teil 3.4.1, p. 1-23.

STEFANOVITS, P. (1971): Bodengeographische Regelmäßigeiten im Donaueinzugsgebiet. Geoforum, 2 (2): 41-46. 